\title{
High-throughput sequencing to identify miRNA biomarkers in colorectal cancer patients
}

\author{
YAN SUN ${ }^{1}$, LIN WANG $^{2}$, SHENG-CHAO GUO $^{1}$, XIAO-BING WU ${ }^{3}$ and XUE-HU XU ${ }^{3}$ \\ ${ }^{1}$ Department of Gastroenterology, The Third Affiliated Hospital of Guangzhou Medical University; \\ ${ }^{2}$ Department of Oncology, Guangzhou Red Cross Hospital; ${ }^{3}$ Department of General Surgery, \\ The Third Affiliated Hospital of Guangzhou Medical University, Guangzhou, Guangdong 510150, P.R. China
}

Received July 26, 2013; Accepted January 30, 2014

DOI: $10.3892 / \mathrm{ol} .2014 .2215$

\begin{abstract}
The altered expression of microRNAs (miRNAs) is associated with a number of cancer types. The study of the association between the miRNA profile and cancer may be useful to identify potential biomarkers of certain types of cancer. In the present study, 19 miRNAs were identified by high-throughput sequencing in the serum of colorectal cancer (CRC) patients. A network analysis was performed based on a computational approach to identify associations between CRC and miRNAs. The present study may be useful to identify cancer-specific signatures and potentially useful biomarkers for the diagnosis of CRC. The network analysis of miRNA-target genes may aid in identifying altered miRNA regulatory networks that are involved in tumor pathogenesis.
\end{abstract}

\section{Introduction}

Colorectal cancer (CRC) is an important contributor to cancer-related mortality and morbidity. Accumulated data has uncovered several critical genes and pathways important in the initiation and progression of CRC (1-3). Large-scale sequencing analyses have identified numerous recurrently mutated genes and chromosomal translocations (4-6). In addition, a number of microRNAs (miRNAs) have been previously reported to be associated with $\operatorname{CRC}(7,8)$. However, how miRNA changes contribute to colorectal tumorigenesis has not yet been defined. Further insight into these changes may identify potential biomarkers or therapeutic targets.

miRNAs are small non-protein coding RNA molecules that regulate gene expression $(9,10)$. miRNAs are important in crucial cellular processes, including development,

Correspondence to: Dr Xue-Hu Xu, Department of General Surgery, The Third Affiliated Hospital of Guangzhou Medical University, 63 Duobao Road, Guangzhou, Liwan, Guangdong 510150, P.R. China

E-mail: xuehuxu@163.com

Key words: colorectal cancer, high-throughput sequencing, miRNA, biomarker differentiation, proliferation, apoptosis and metabolism $(11,12)$. miRNAs have been proven to interact with potential oncogenes or tumor suppressors, and a number of miRNAs are differentially expressed in normal and neoplastic tissues and in tumors. The differential expression of miRNA has been previously evaluated as a predictive signature of cancer (13-17).

The present study investigated the expression profile of miRNAs in the serum of CRC patients. In total, 19 miRNAs were identified by high-throughput sequencing. A network analysis was performed based on a computational approach to identify associations between CRC and miRNAs. The network analysis of miRNA-target genes may aid in identifying altered miRNA regulatory networks that are involved in tumor pathogenesis.

\section{Materials and methods}

Samples and RNA extraction. Primary tumor and neighboring non-tumorous tissues were obtained from five CRC patients. All samples were collected according to procedures approved by the Institutional Review Board of the Guangzhou Medical University (Guangzhou, China) and individuals can not be identified from data or images included in the present study.

Tissue samples were flash frozen in liquid nitrogen and stored at $-80^{\circ} \mathrm{C}$ until nucleic acid extraction. In total, $200 \mathrm{mg}$ of fresh frozen tissues were used to isolate total RNA by phenol extraction (TRIzol reagent; Invitrogen, Life Technologies, Carlsbad, CA, USA). RNA concentration and purity were controlled by A NanoDrop spectrophotometer (Thermo Fisher Scientific, Waltham, MA, USA) . The Agilent 2100 Bioanalyzer (Agilent Technologies, Inc., Santa Clara, CA, USA) was used to measure the quantity, integrity and purity of the small RNA. Patients provided written informed consent and the study was approved by the ethics committee of the Third Hospital of the Guangzhou Medical University.

miRNA sequencing and sequence analysis. The miRNA expression profile was determined using the Ion Torrent PGM $^{\text {тм }}$ sequencer (Life Technologies). Briefly, the Ion Total RNA-Seq kit v2 (Life Technologies) was used to make small RNA libraries that preserve strand information. The templates were prepared using the Ion OneTouch ${ }^{\mathrm{TM}}$ system (Life Technologies), and small RNA analysis was performed 
Kyoto Encyclopedia of Genes and Genomes, a collection of online databases. The Pathway Database records networks of molecular interactions in cells and their variants.

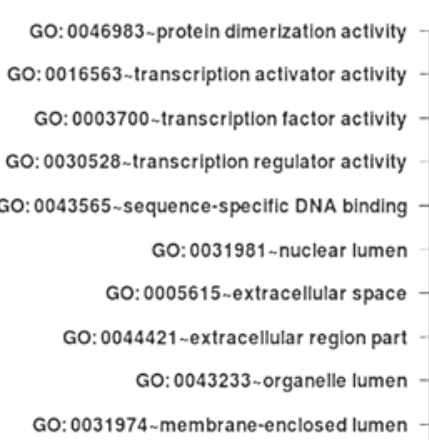

GO: 0010557 positive regulation of macromolecule blosynthetic process GO: 0031328 positive regulation of cellular blosynthetic process GO: 0010604 positive regulation of macromolecule metabollc process GO: 0009891 positive regulation of blosynthetic process GO: 0042127 regulation of cell proliferation

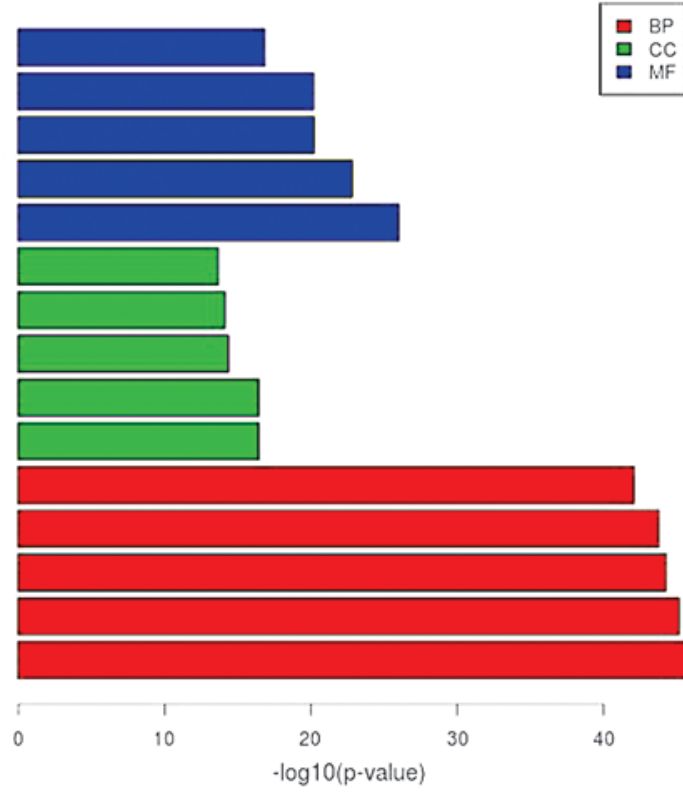

Figure 1. GO analysis. A number of target genes were found to be involved in cell proliferation and apoptosis. BP, biological process; CC, celluar component; MF, molecular function; GO, gene ontology.



Figure 2. Pathway analysis. Transforming growth factor $\beta$ and Toll-like signal pathways were found to be involved in cell proliferation and apoptosis.

using the Ion PGM ${ }^{\mathrm{TM}}$ sequencer. The miRNA sequences were analyzed by the Torrent Suite software (Life Technologies), and by miRWalk (http://mirwalk.uni-hd.de) and miRBase (http://www.mirbase.org). In addition, gene ontology (GO) and pathway analyses were performed using several tools that identify pathways and GO based on data sets from sequencing with the intent to identify miRNA-related genes and pathways. The tools include Ingenuity Systems Bioinformatics Software (Ingenuity Systems, Inc., Redwood City, CA, USA), FatiGO (http://fatigo.bioinfo.cnio.es) and Gene Expression Omnibus (http://www.ncbi.nlm.nih.gov/gds).

\section{Results}

Altered miRNA expression in CRC patients. The miRNA profiles of five pairs of solid tumor and adjacent tissues were compared. In total, 16 miRNAs exhibited higher expression in the solid tumor tissues, while three miRNAs exhibited higher 
Table I. Identification of miRNAs with higher expression profiles in normal or solid tumor tissues.

\begin{tabular}{lr}
\hline Solid tumor tissues & Normal adjacent tissues \\
\hline hsa-miR-135b-5p & hsa-miR-100-5p \\
hsa-miR-146a-5p & hsa-miR-138-5p \\
hsa-miR-148b-3p & \\
hsa-miR-17-5p & \\
hsa-miR-196a-5p & \\
hsa-miR-200a-3p & \\
hsa-miR-20a-5p & \\
hsa-miR-21-5p & \\
hsa-miR-223-3p & \\
hsa-miR-27a-5p & \\
hsa-miR-29b-3p & \\
hsa-miR-30e-5p & \\
hsa-miR-374b-5p & \\
hsa-miR-4787-5p \\
hsa-miR-485-3p \\
hsa-miR-660-5p
\end{tabular}

miR/miRNA, microRNA; CRC, colorectal cancer.

expression profiles in the normal tissues; these are listed in Table I.

GO and pathway analyses of miRNA target genes. The results of the GO analysis showed that a number of target genes are involved in cell proliferation and apoptosis. The pathway analysis showed that the transforming growth factor $\beta$ and Toll-like signal pathways are also involved (Fig. 1 and 2).

\section{Discussion}

miRNAs are small non-coding RNAs that enhance the cleavage or translational repression of specific mRNAs with recognition site(s) in the 3 '-untranslated region. Since the identification of the miRNAs, several large-scale studies have compared the profiles of miRNA expression patterns between non-tumor and tumor tissues $(18,19)$. A number of lines of evidence have shown that miRNA expression is predictive of outcome in patients with solid tumors. In lung cancer, low levels of let-7a have been associated with a short survival time following surgery. In addition, previous miRNA microarray expression profiling of tumors and paired non-tumorous tissues has been performed in colon cancer patients to identify miRNA expression patterns associated with outcome, and high levels of miR-21 have been found to be associated with a short overall survival time, independent of other factors $(19,20)$.

In the current study, 19 miRNAs were identified by high-throughput sequencing. A network analysis was performed based on a computational approach to identify associations between CRC and miRNAs. The current study may be useful to identify cancer-specific signatures and potentially useful biomarkers for the diagnosis of CRC. The network analysis of miRNA-target genes may aid in identifying altered miRNA regulatory networks that are involved in tumor pathogenesis.

\section{Acknowledgements}

The present study was supported by the Science and Information Technology Foundation of Guangzhou (grant nos. 2010J-E141 and 2011J4100051), the Guangdong Provincial Science and Technology Department (grant no. 2011B0904005260) and the Natural Science Foundation of Guangdong Province (grant no. S2012040006707).

\section{References}

1. Tjandra JJ, Kilkenny JW, Buie WD, et al; Standards Practice Task Force; American Society of Colon and Rectal Surgeons: Practice parameters for the management of rectal cancer (revised). Dis Colon Rectum 48: 411-423, 2005.

2. Fearon ER: Molecular genetics of colorectal cancer. Annu Rev Pathol 6: 479-507, 2011.

3. Aaltonen LA, Peltomäki P, Leach FS, et al: Clues to the pathogenesis of familial colorectal cancer. Science 260: 812-816, 1993.

4. Bass AJ, Lawrence MS, Brace LE, et al: Genomic sequencing of colorectal adenocarcinomas identifies a recurrent VTI1A-TCF7L2 fusion. Nat Genet 43: 964-968, 2011.

5. Sjöblom T, Jones S, Wood LD, et al: The consensus coding sequences of human breast and colorectal cancers. Science 314: 268-274, 2006.

6. Wood LD, Parsons DW, Jones S, et al: The genomic landscapes of human breast and colorectal cancers. Science 318: 1108-1113, 2007.

7. Schepeler T, Reinert JT, Ostenfeld MS, et al: Diagnostic and prognostic microRNAs in stage II colon cancer. Cancer Res 68: 6416-6424, 2008.

8. Schetter AJ, Leung SY, Sohn JJ, et al: MicroRNA expression profiles associated with prognosis and therapeutic outcome in colon adenocarcinoma. JAMA 299: 425-436, 2008.

9. Lim LP, Glasner ME, Yekta S, Burge CB and Bartel DP: Vertebrate microRNA genes. Science 299: 1540, 2003.

10. Zeng Y and Cullen BR: Sequence requirements for micro RNA processing and function in human cells. RNA 9: 112-123, 2003.

11. Ambros V: The functions of animal microRNAs. Nature 431: 350-355, 2004

12. Kloosterman WP and Plasterk RH: The diverse functions of microRNAs in animal development and disease. Dev Cell 11: 441-450, 2006.

13. Perera RJ and Ray A: MicroRNAs in the search for understanding human diseases. BioDrugs 21: 97-104, 2007.

14. Iorio MV, Visone R, Di Leva G, et al: MicroRNA signatures in human ovarian cancer. Cancer Res 67: 8699-8707, 2007.

15. Caldas $C$ and Brenton JD: Sizing up miRNAs as cancer genes. Nat Med 11: 712-714, 2005.

16. Jones KB, Salah Z, Del Mare S, et al: miRNA signatures associate with pathogenesis and progression of osteosarcoma. Cancer Res 72: 1865-1877, 2012.

17. Volinia S, Galasso M, Costinean S, et al: Reprogramming of miRNA networks in cancer and leukemia. Genome Res 20: 589-599, 2010.

18. Lu J, Getz G, Miska EA, et al: MicroRNA expression profiles classify human cancers. Nature 435: 834-838, 2005.

19. Luo X, Burwinkel B, Tao S and Brenner H: MicroRNA signatures: novel biomarker for colorectal cancer? Cancer Epidemiol Biomarkers Prev 20: 1272-1286, 2011.

20. Jeong HC, Kim EK, Lee JH, Lee JM, Yoo HN and Kim JK: Aberrant expression of let-7a miRNA in the blood of non-small cell lung cancer patients. Mol Med Rep 4: 383-387, 2011. 